\title{
Impact of discrete wavelet transform on discriminating airborne hyperspectral tropical rainforest tree species
}

\begin{abstract}
Discriminating tropical rainforest tree species is still a challenging task due to a variety of species with high spectral similarity and due to very limited studies conducted in this area. We are investigating the effect of discrete wavelet transform (DWT) on enhancing discrimination of tropical rainforest tree species. For this purpose, airborne imaging spectrometer for applications (AISA) airborne hyperspectral data obtained from Malaysianô rainforest area are used; six tree species were selected from the study area. For comparison purposes, the performance of DWT is compared with the original reflectance, first, and second derivative spectra by using five different spectral measure techniques. An overall discrimination accuracy of $\sim 74 \%$ is obtained with DWT using Euclidean distance, which outperforms the original reflectance and first and second derivatives by $\sim 16.6,11.9$, and $22.1 \%$, respectively. The results suggest a significant impact of the DWT approach on improving tropical rainforest tree species discrimination.
\end{abstract}

Keyword: Airborne hyperspectral remote sensing; Tropical rainforest; Tree species discrimination; Discrete wavelet transform; Spectral dimensionality; Derivative spectra 